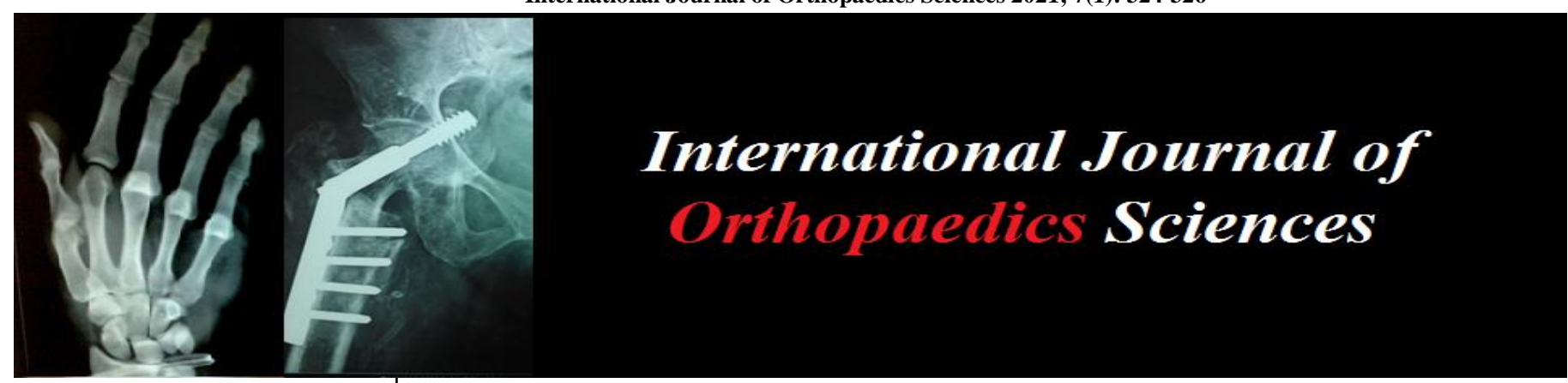

E-ISSN: 2395-1958

P-ISSN: 2706-6630

IJOS 2021; 7(1): 324-326

(C) 2021 IJOS

www.orthopaper.com

Received: 04-11-2020

Accepted: 09-12-2020

Dr. Prabhakar TS

MBBS, D'Ortho, M.S.

(Orthopaedics) Senior Specialist in Orthopaedics and Orthopaedic

Surgeon at K.C. General

Hospital Malleswaram,

Bangalore, Karnataka, India

\section{Post- operative complications for patients with Rheumatoid arthritis undergoing elective orthopedic procedures with or without methotrexate}

\section{Dr. Prabhakar TS}

DOI: https://doi.org/10.22271/ortho.2021.v7.i1f.2503

\section{Abstract}

Background: Rheumatoid arthritis (RA) is a common and disabling condition that is now often treated by the cytotoxic drug methotrexate. The present study was conducted to assess post- operative complications for patients with Rheumatoid arthritis undergoing elective orthopedic procedures with or without methotrexate.

Materials \& Methods: 60 patients diagnosed with Rheumatoid arthritis were divided into 2 groups of 30 each. Group I patients with RA who were receiving methotrexate for at least six weeks before surgery and in whom methotrexate treatment was not discontinued. Group II patients with RA who were receiving methotrexate for type of surgery and in whom methotrexate treatment was stopped two weeks before surgery and restarted two weeks after surgery. Group III patients with RA who underwent elective orthopaedic surgery during the study period and who had not received methotrexate treatment. Complications were recorded.

Results: Group I had 11 males and 9 females, group II had 10 males and 10 females and group III had 8 males and 12 females. Mean age was 54.2 years, 56.7 years and 54.6 years respectively, disease duration was 18.2 years, 19.1 years and 19.8 years respectively, baseline articular index was 14.1 years, 16 years and 15.7 years respectively and HAQ was $1.8,1.8$ and 1.7 years respectively. The most common complication was dehiscence seen 7 in group III, 3 in group II and 1 in group I followed by discharge seen 7 in group III, 2 in group II and 1 in group I. The difference was significant $(P<0.05)$.

Conclusion: Continuation of methotrexate treatment does not increase the risk of either infections or of surgical complications occurring in patients with RA.

Keywords: Methotrexate, Rheumatoid arthritis, Surgical

\section{Introduction}

Rheumatoid arthritis (RA) is a common and disabling condition that is now often treated by the cytotoxic drug methotrexate. Once the inflammation of rheumatoid disease is controlled by methotrexate, if the drug is suddenly stopped the rheumatoid disease often flares, thus making movement painful and rehabilitation and mobilisation after any surgical procedure more difficult ${ }^{[1]}$.

Orthopaedic procedures have substantially improved the overall function and quality of life in RA patients ${ }^{[2]}$. However, complications after surgery, especially post-operative surgical site infections (SSIs), pose serious functional and psychological disadvantages during the course of RA treatment. Usually, this RA patient population has a higher baseline risk of infectious diseases compared with the general population ${ }^{[3]}$. Therefore, the infection rate after surgery in RA patients could be even higher, although the data are inconsistent, and these infrequent complications in joint surgery are seen in only a small percentage of patients. Total joint replacement is a common procedure in RA, and is one of the most successful surgical interventions for RA that reduces pain and enhances physical function ${ }^{[4]}$.

Prospective studies subsequently compared the risk of postoperative surgical complications in a further 313 procedures in patients with RA who received methotrexate treatment within four weeks of elective orthopaedic surgery and 176 patients with RA who did not receive methotrexate within four weeks of surgery. 22 of $332(7 \%)$ procedures in subjects who continued methotrexate developed early postoperative complications, compared with 10 of 210
Corresponding Author: Dr. Prabhakar TS MBBS, D'Ortho, MS. (Orthopaedics) Senior Specialist in Orthopaedics and Orthopaedic Surgeon at K.C. General

Hospital Malleswaram,

Bangalore, Karnataka, India 
(5\%) procedures on patients with RA who did not receive methotrexate treatment before surgery-that is, the overall results showed a trend for an increased risk of early postoperative surgical complications in subjects who continued methotrexate before surgery ${ }^{[5,6]}$. The present study was conducted to assess post- operative complications for patients with Rheumatoid arthritis undergoing elective orthopedic procedures with or without methotrexate.

\section{Materials and Methods}

The present study comprised of 60 patients diagnosed with Rheumatoid arthritis of both genders. All patients were informed regarding the study and their written consent was obtained.

Data such as name, age, gender etc. was recorded. Patients were divided into 2 groups of 30 each. Group I patients with RA who were receiving methotrexate for at least six weeks before surgery and in whom methotrexate treatment was not discontinued. Group II patients with RA who were receiving methotrexate for type of surgery and in whom methotrexate treatment was stopped two weeks before surgery and restarted two weeks after surgery. Group III patients with RA who underwent elective orthopaedic surgery during the study period and who had not received methotrexate treatment. Complications such as reddening of wound, discharge from wound, systemic infection, or wound dehiscence, loosening of implants, or any complication requiring a secondary revision procedure and occurring within one year of surgery were recorded. Results were analysed statistically. $\mathrm{P}$ value less than 0.05 was considered significant.

\section{Results}

Table 1: Assessment of parameters

\begin{tabular}{|c|c|c|c|c|}
\hline Parameters & Group I & Group II & Group III & P value \\
\hline M:F & $11: 9$ & $10: 10$ & $8: 12$ & 0.12 \\
\hline Mean age & 54.2 & 56.7 & 54.6 & 0.15 \\
\hline Disease duration & 18.2 & 19.1 & 19.8 & 0.21 \\
\hline Baseline articular index & 14.1 & 16 & 15.7 & 0.18 \\
\hline HAQ & 1.8 & 1.8 & 1.7 & 0.95 \\
\hline
\end{tabular}

Table I shows that group I had 11 males and 9 females, group II had 10 males and 10 females and group III had 8 males and 12 females. Mean age was 54.2 years, 56.7 years and 54.6 years respectively, disease duration was 18.2 years, 19.1 years and 19.8 years respectively, baseline articular index was 14.1 years, 16 years and 15.7 years respectively and HAQ was $1.8,1.8$ and 1.7 years respectively. The difference was non- significant $(\mathrm{P}>0.05)$.

Table 2: Surgical procedures performed in all groups

\begin{tabular}{|c|c|c|c|c|}
\hline Parameters & Group I & Group II & Group III & P value \\
\hline Shoulder & 5 & 2 & 4 & 0.11 \\
\hline Wrist & 4 & 5 & 4 & 0.17 \\
\hline Elbow & 6 & 6 & 5 & 0.18 \\
\hline Hip & 3 & 3 & 4 & 0.19 \\
\hline Ankle & 2 & 4 & 3 & 0.21 \\
\hline
\end{tabular}

Table II, graph I shows that surgical procedures were performed in shoulder 5 in group I, 2 in group II and 4 in group III, wrist in 4, 5 and 4 in group I, II and III respectively, elbow in 6, 6 and 5 in group I, II and III respectively, hip in 3, 3 and 4 in group I, II and III respectively and ankle in 2, 4 and 3 in group I, II and III respectively. The difference was nonsignificant $(P>0.05)$.

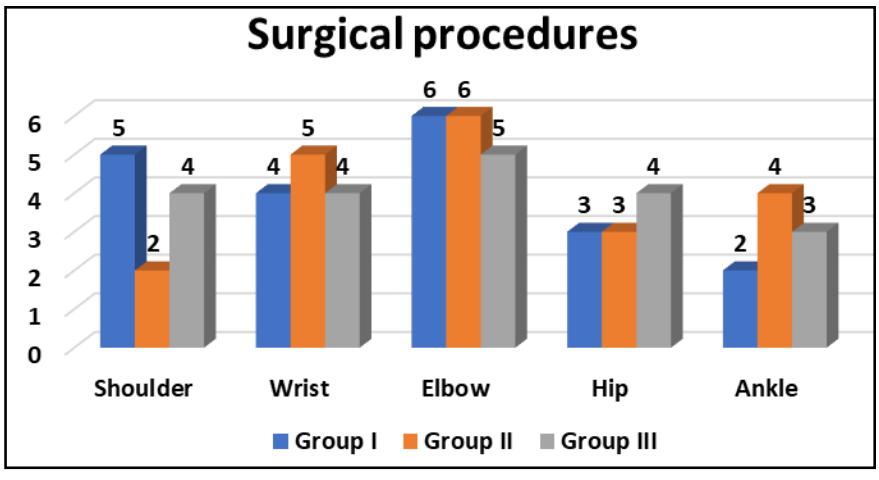

Graph 1: Surgical procedures performed in all groups

Table 3: Comparison of complications

\begin{tabular}{|c|c|c|c|c|}
\hline Complications & Group I & Group II & Group III & P value \\
\hline Rubor & 1 & 3 & 5 & 0.05 \\
\hline Discharge & 1 & 2 & 6 & 0.02 \\
\hline Dehiscence & 1 & 3 & 7 & 0.04 \\
\hline Discharge & 1 & 2 & 7 & 0.02 \\
\hline Total & 4 & 10 & 25 & \\
\hline
\end{tabular}

Table III, graph II shows that most common complication was dehiscence seen 7 in group III, 3 in group II and 1 in group I followed by discharge seen 7 in group III, 2 in group II and 1 in group I. The difference was significant $(\mathrm{P}<0.05)$.

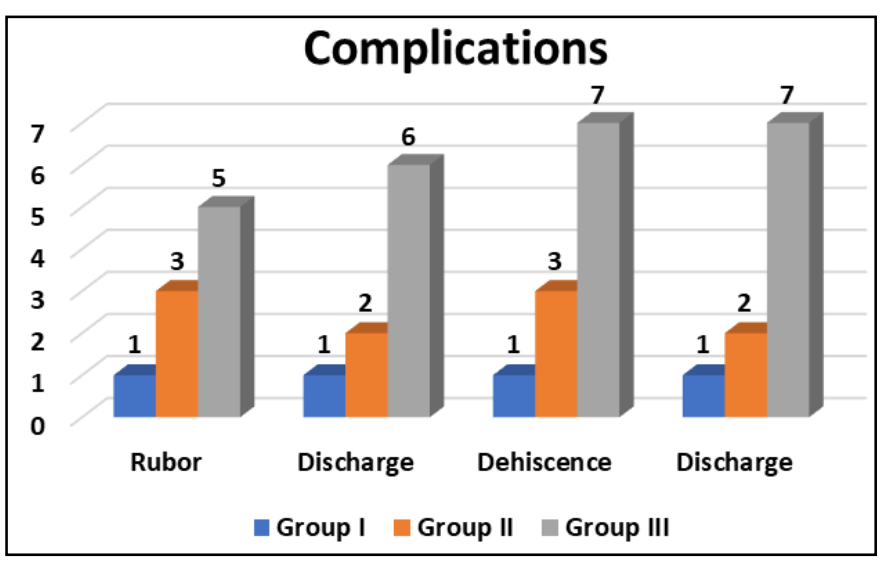

Graph 2: Comparison of complications

\section{Discussion}

Rheumatoid arthritis is a disabling inflammatory condition which is treated with methotrexate, though there are many new drugs currently used in addition or in place of methotrexate ${ }^{[7]}$. There have been studies indicating that continuation of methotrexate during the intraoperative phase causes complications including infection. There were additional prospective and retrospective studies confirming this finding ${ }^{[8]}$. These studies led to the practice of stopping methotrexate weeks prior to surgery and restarting after surgery. unfortunately, this led to aggravation of the rheumatoid symptoms after surgery ${ }^{[9]}$. The present study was conducted to assess post- operative complications for patients with Rheumatoid arthritis undergoing elective orthopedic procedures with or without methotrexate.

In present study, group I had 11 males and 9 females, group II had 10 males and 10 females and group III had 8 males and 12 females. Mean age was 54.2 years, 56.7 years and 54.6 years respectively, disease duration was 18.2 years, 19.1 years and 19.8 years respectively, baseline articular index was 14.1 years, 16 years and 15.7 years respectively and HAQ was $1.8,1.8$ and 1.7 years respectively. Sreekumar et al. ${ }^{[10]}$ 
carried out a controlled study of complications after elective surgery in rheumatoid arthritis (RA) patients who either continued or discontinued methotrexate prior to surgery. In this study they showed that continuation of methotrexate therapy prior to orthopaedic surgery did not increase the risk of infection or surgical complication occurring in patients with RA within one year of surgery. Thirty-one were fully assessed in clinic and 34 underwent a structured telephone interview. There were no incidences of deep bone infection in any patient group so that there is no evidence that continued methotrexate therapy in the perioperative period increases the risk of late deep infections.

We found that surgical procedures were performed in shoulder 5 in group I, 2 in group II and 4 in group III, wrist in 4,5 and 4 in group I, II and III respectively, elbow in 6, 6 and 5 in group I, II and III respectively, hip in 3, 3 and 4 in group I, II and III respectively and ankle in 2, 4 and 3 in group I, II and III respectively. Grenan et al ${ }^{[11]}$ conducted a prospective randomised study of postoperative infection or surgical complications occurring within one year of surgery in patients with RA who underwent elective orthopaedic surgery. 388 patients with RA who were to undergo elective orthopaedic surgery. Patients who were receiving methotrexate were randomly allocated to groups who either continued methotrexate (group A) or who discontinued methotrexate from two weeks before surgery until two weeks after surgery (group B). Their complication rates were compared with complications occurring in 228 patients with RA (group C) who were not receiving methotrexate and who also underwent elective orthopaedic surgery. Signs of postoperative infection were recorded, including rubor, discharge, systemic infection, and frequency of wound dehiscence as well as the incidence of any surgical complication requiring a secondary revision procedure that occurred within one year of surgery. The frequencies of flare up activity of RA at six weeks and six months after surgery were also recorded. A flare of rheumatoid disease was defined as an increase in joint pain in two or more joints notified by the patient as well as by an increase in articular index of at least $25 \%$ after surgery. Signs of infection or surgical complications occurred in two of 88 procedures in group A $(2 \%), 11$ of 72 procedures in group B (15\%), and 24 of $228(10.5 \%)$ procedures in group C. The surgical complication or infection frequency in group A was less than that in either group $B(p<0.003)$ or group $C$ $(p=0.026)$. At six weeks after surgery there were no flares in group A, six flares in group B (8\%), and six flares in group C (2.6\%). Logistic regression analysis of the overall surgical complication rate in all the patients with RA studied showed that methotrexate, whether continued or discontinued before surgery, did not increase the early complication rate in the patients with RA who underwent elective orthopaedic surgery.

We found that most common complication was dehiscence seen 7 in group III, 3 in group II and 1 in group I followed by discharge seen 7 in group III, 2 in group II and 1 in group I. The shortcoming of the study is small sample size.

\section{Conclusion}

Present study suggested that continuation of methotrexate treatment does not increase the risk of either infections or of surgical complications occurring in patients with RA.

\section{References}

1. Bridges S, Lopez Mendez A, Han K, Tracy F, Alarson G. Should methotrexate be discontinued before elective orthopaedic surgery in patients with rheumatoid arthritis? J Rheumatol 1991;18:984-8.

2. Kasden M, June L. Post-operative results of rheumatoid arthritis patients on methotrexate at the time of reconstructive surgery of the hand. Orthopaedics 1993;16:1233-5.

3. Sany J, Anaya J, Canovas F, Combe B, Jorgensen C, Saker S, et al. Influence of methotrexate and the frequency of postoperative infections/complications in patients with rheumatoid arthritis. J Rheumatol 1993;20:1129-32.

4. Perhala R, Wilke W, Clough J, Segal A. Local infectious complications following large joint replacements in rheumatoid arthritis patients treated with methotrexate versus those not treated with methotrexate. Arthritis Rheum 1991;34:146-52.

5. Carpenter M, West S, Jones D. Post-operative joint infections in rheumatoid arthritis patients on methotrexate therapy. Othopaedics 1996;19:207-10.

6. Richie D, Boyle JA, McInnes T, Jasani M, Dalakos T, Grieveson $\mathrm{P}$, et al. Clinical trials with an articular index on the assessment of joint tenderness in patients with rheumatoid arthritis. Q J Med 1958;37:393-406.

7. Fries JF, Spitz P, Kraines RG, Holman HR. Measurement of patient outcome in arthritis. Arthritis Rheum 1980;23:1327-45.

8. Garvin K, Nebrasha O, Hanssen A. Infection after total hip arthroplasty. J Bone Joint Surg Am 1995;77:1576-87.

9. O'Callaghan J, Forrest M, Brooks P. Inhibition of neutrophil chemo-taxis in methotrexate treated rheumatoid arthritis patients. Rheumatol Int 1988;8:41-5.

10. Sreekumar R, Gray J, Kay P, Grennan DM. Methotrexate and post- operative complications in patients with rheumatoid arthritis undergoing elective orthopaedic surgery-A ten years follow-up. Acta Orthopaedica Belgica 2011;77(6):823.

11. Grennan DM, Gray J, Loudon J, Fear S. Methotrexate and early postoperative complications in patients with rheumatoid arthritis undergoing elective orthopaedic surgery. Annals of the rheumatic diseases 2001;60(3):214-7. 